\title{
Changes in the Concentrations of Corticoid Metabolites - The Effect of Stress, Diet and Analytical Method
}

\author{
Michaela Dušková, Lucie Sosvorová, Jana Vítků, Hana Jandíková, \\ Beáta Rácz, Tereza Chlupáčová, Jose De Cordeiro, Luboslav Stárka \\ Institute of Endocrinology, Prague, Czech Republic
}

Received September 29, 2015; Accepted November 16, 2015.

Key words: Cortisol - Cortisone - Aldosterone - Corticosterone - Stress Food intake

\begin{abstract}
A precisely measured corticoid level is important for decision-making in daily clinical practice. These levels can be influenced in the pre-analytical phase, when the effect of stress, timing, and diet can be important. The aim of this study was to elucidate optimal conditions for blood sampling as well as the choice of analytical methods, which they will be used in measuring of corticoids. By studying ten women, we focused on the influences of the stress of cannulation and a large lunchtime meal on cortisol, cortisone, aldosterone and corticosterone levels. We further compared results of cortisol measurements from RIA and LC-MS/MS. Stress from cannulation caused increase of cortisol, cortisone and corticosterone already, when the cannula was being inserted. This indicates that this increase is stimulated by fear of the blood withdrawing rather, than just by the needle insertion itself. The effect of stress on corticosterone disappeared after an hour, while effect on other corticoids was still apparent. Concerning the lunchtime meal, we found an increase in all measured corticoids between 11 and 12 o'clock. After the food, there were marked decreases in cortisone and aldosterone, while declining levels of cortisol and corticosterone had rather plateaus. We compared cortisol in 90 plasma samples measured by a commercial RIA kit and the LC-MS/ MS method. Results from both methods showed a strong correlation $(r=0.85)$. When measuring corticoid metabolites, the chosen analytical method, eliminating stress factors, and precisely timed blood sampling considering the daily rhythm and food intake are critical.
\end{abstract}

This study was supported by grant IGA MZ ČR number 12340-5.

Mailing Address: Michaela Dušková, MD., PhD., Institute of Endocrinology, Národní 8, 11694 Prague 1, Czech Republic; Phone: +420 224905 412; e-mail: mduskova@endo.cz 


\section{Introduction}

Measuring plasma cortisol levels is a common task of clinical biochemistry laboratories, and not just when ordered by endocrinologists. However, in the pre-analytical phase there are various influences that can distort the information sought. These influences include stress brought about by the sampling, poorlytimed sampling, not taking the sampling after fasting, and choosing a method that is incompatible with the desired aims. Errors in the pre-analytical phase significantly complicate the interpretation of results, and could lead to an incorrect diagnosis.

Cortisol has a marked circadian rhythm in secretion that consists of a decline before bedtime followed by a maximum decline at midnight to under $100 \mathrm{nmol} / \mathrm{l}$, and then an increase starting two hours before waking. Later during the day cortisol again declines and the cycle repeats. The basis of cortisol circadian rhythm is formed by episodic pulses occurring with almost hourly frequency - an ultradian rhythm. Changes to cortisol levels as part of the circadian rhythm are based on changes in the amplitude of these ultradian pulses (Lightman and ConwayCampbell, 2010).

There is a significant rise in cortisol in the first hour after waking, and these higher levels are the basis for the cortisol awakening response (CAR). Both serum and plasma cortisol levels increase by about $50-70 \%$ during the first 30 minutes after waking and remain increased for about 60 minutes (Pruessner et al., 1997). For this reason, if not specifically designed to analyse CAR then sampling for measuring cortisol levels should be performed at least one hour after waking.

In addition to the morning rise in cortisol, slight increases in cortisol levels associated with food have been described. We have performed two studies focused on the influence of daily timing and food intake on steroid hormone levels. In the first study we mapped changes in steroid hormones in relation to the time of day and regular food intake over 16 hours. We found decrease of cortisol levels after the main meals, which was probably due to withdrawals hours apart from meals (Rácz et al., 2015a), so we made a second more detailed study.

We tested this influence of food intake on cortisol levels in a second study, analysing levels after various forms of stimulation. In addition to a standard breakfast we compared levels after oral (OGTT) and intravenous (IVGTT) glucose tests as well as after ingesting psyllium as a model of the mechanical stimulation of the gastrointestinal tract (Rácz et al., 2015b). Using identical analytical conditions, we found differences in the trends in cortisol levels after these different food stimuli, but levels after 120 minutes were all the same. There was a decrease in the physiological decline of cortisol levels after each of the stimuli except psyllium. This decrease was most marked after IVGTT, lasting up to 60 minutes. After OGTT and IVGTT there was also a plateau in cortisol levels. After the standard breakfast there was an increase in cortisol levels after 40 minutes (Rácz et al., 2015b).

The aim of this present study was to expand on those findings in order to determine the optimal conditions for cortisol sampling as well as the choice of 
analytical method. Firstly, we analysed the influence of stress due to cannulation on cortisol levels. Next, we focused on a detailed mapping of changes around the time of the main meal of the day, which in our country is lunchtime. Then, we compared measurements of cortisol using two analytical methods, radioimmunoassay (RIA) and liquid chromatography with tandem mass spectrometry (LC-MS/MS).

The newly-developed LC-MS/MS method allows multiple steroids to be measured at one time. We therefore expanded the study to analyse not just cortisol but all main corticoids after stress brought on by cannulation. We measured the main glucocorticoids, cortisol and its metabolite cortisone, plus the main mineralocorticoids, aldosterone and its precursor corticosterone, which is a minor steroid in humans.

\section{Material and Methods}

The study was performed using 10 healthy women of reproductive age in the follicular phase of their cycle (days 1-7 after menstruation). The average age was $33.6 \pm 2.56$ years and average BMI $25.06 \pm 1.3$. The women had no chronic diseases, were non-smokers, and did not use hormonal contraceptives or any other medications. Before starting the study, they were advised to maintain a balanced regimen of 8 hours of sleep, regular eating according to a recommended menu, and restrain from consuming alcohol. All participants were given explanations about the study and signed informed consent. The study was approved by the ethical commission of the Institute of Endocrinology.

Each woman passed two tests during two consecutive menstrual cycles:

\section{1) Stimulation test by stress and food}

Each participant woke up at 6:30 in the morning. They had at 7:00 standard breakfast (two slices of bread, $50 \mathrm{~g}$ of breast-meat chicken slices, 1 slice of fresh cheese; total caloric content of the breakfast was $515 \mathrm{kcal}$, total protein content: $20.58 \mathrm{~g}$, total carbohydrates: $47.75 \mathrm{~g}$, total fat: $24.9 \mathrm{~g}$ ). The test started at 10:00, when cannula was inserted into the forearm or cubital vein. The time schedule - according to which the blood was drawn - was: 10:00, 10:15, 10:30, 10:45 and 11:00.

After the blood sampling at 12 o'clock, the participants of this part of the study received lunch (beef broth soup, turkey, potato dumplings, and sauerkraut; total content of the lunch was $679 \mathrm{kcal}$, total protein content: $45.55 \mathrm{~g}$, total carbohydrates: $100.4 \mathrm{~g}$, total fat: $11.5 \mathrm{~g}$ ). The lunch was followed by blood drawings at $12: 30,13: 00$ and 13:30.

\section{2) Blank test}

Each participant woke up at 6:30 in the morning. They had at 7:00 standard breakfast (two slices of bread, $50 \mathrm{~g}$ of breast-meat chicken slices, 1 slice of fresh cheese; total caloric content of the breakfast was $515 \mathrm{kcal}$, total protein content: 
$20.58 \mathrm{~g}$, total carbohydrates: $47.75 \mathrm{~g}$, total fat: $24.9 \mathrm{~g}$ ). The peripheral cannula was inserted at 7:30 into the forearm or cubital vein. The blood was drawn according to the same schedule as it was in the first part of the study, it means, that at 10:00 was drawn the first blood sample, which was followed by additional samples at 10:15, 10:30, 10:45 and 11:00. In contrast to the stimulation test, in the blank test participants did not have any lunch at 12 o'clock, but they were submitted to samplings similarly as the group in the previous part of the study. The schedule of the blood drawings was: 12:00, 12:30, 13:00 and 13:30.

Blood was taken into a Vacuette Serum Clot Activator tubes (a plastic tube with a clotting activator and separation gel). Serum was obtained by centrifugation for 5 minutes at $2000 \mathrm{~g}$ at $4{ }^{\circ} \mathrm{C}$, and stored at $-20^{\circ} \mathrm{C}$.

Cortisol, cortisone, corticosterone and aldosterone were measured by LC-MS/MS (Sosvorova et al., 2015), and cortisol was additionally measured using an RIA kit from Immunotech (Czech Republic).

\section{Statistical analyses}

The relationships between dependent variables and the effects of sampling time were evaluated using a repeated measures ANOVA model consisting of the following factors:Time (10:00, 10:15, 10:30, 10:45 and 11:00 for experiment 1); (11:00, 12:00, 12:30, 13:00 and 13:30 for experiment 2) and Subject (explaining inter-individual variability). The ANOVA model was followed by least significant difference (LSD) multiple comparisons. To eliminate skewed data distribution and heteroscedasticity, the original data were transformed by Box-Cox transformation to attain a Gaussian distribution and constant variance before further processing. Simple regression was used to compare the two different analytical methods, with the RIA method chosen as the reference since it is commonly used during routine cortisol measurements. The statistical software Statgraphics Centurion, version XV from Statpoint Inc. (Herndon, Virginia, USA) was used for all statistical analyses.

\section{Results}

Stress

Figure 1 shows the profile of cortisol levels when cannulation was being performed as well as when cannulation had been performed 150 minutes before the first blood sampling.Values just after inserting the cannula were significantly higher than values when calm. This reflects the fact that blood drawing can invoke minor or even fairly high stress in some patients. Higher levels of plasma cortisol lasted at least 1 hour after the first sampling. These results indicate that merely the knowledge that blood will be drawn can stimulate higher cortisol levels, not just the needle insertion itself. Cortisone levels were also increased, similarly as for cortisol (Figure 1). 

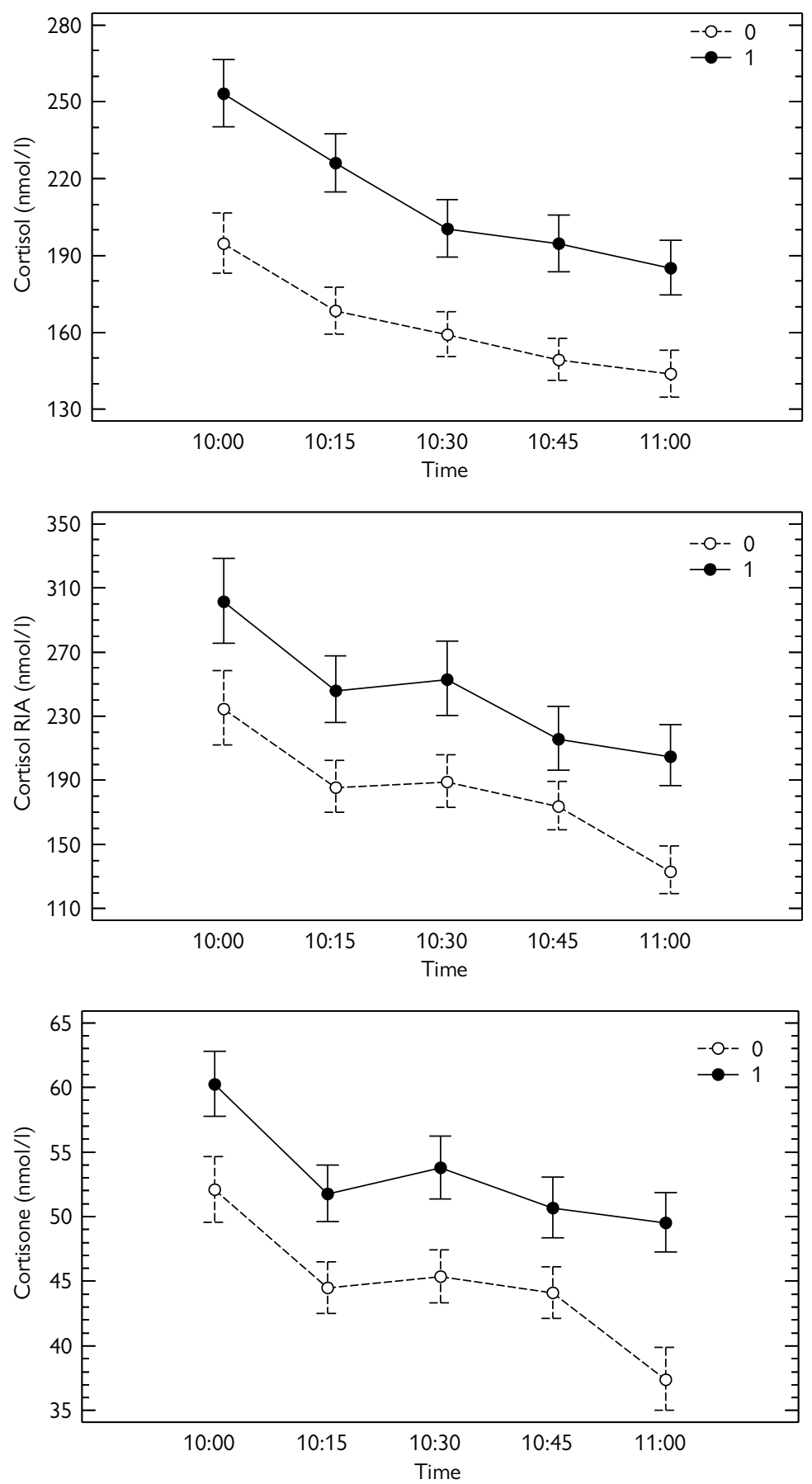

Figure 1 - Influence of the time of blood drawing after cannulisation on cortisol levels (measured LC-MSIMS and RIA) and cortisone (0 - cannula inserted at 8:00; 1 - cannula inserted at 10:00). 

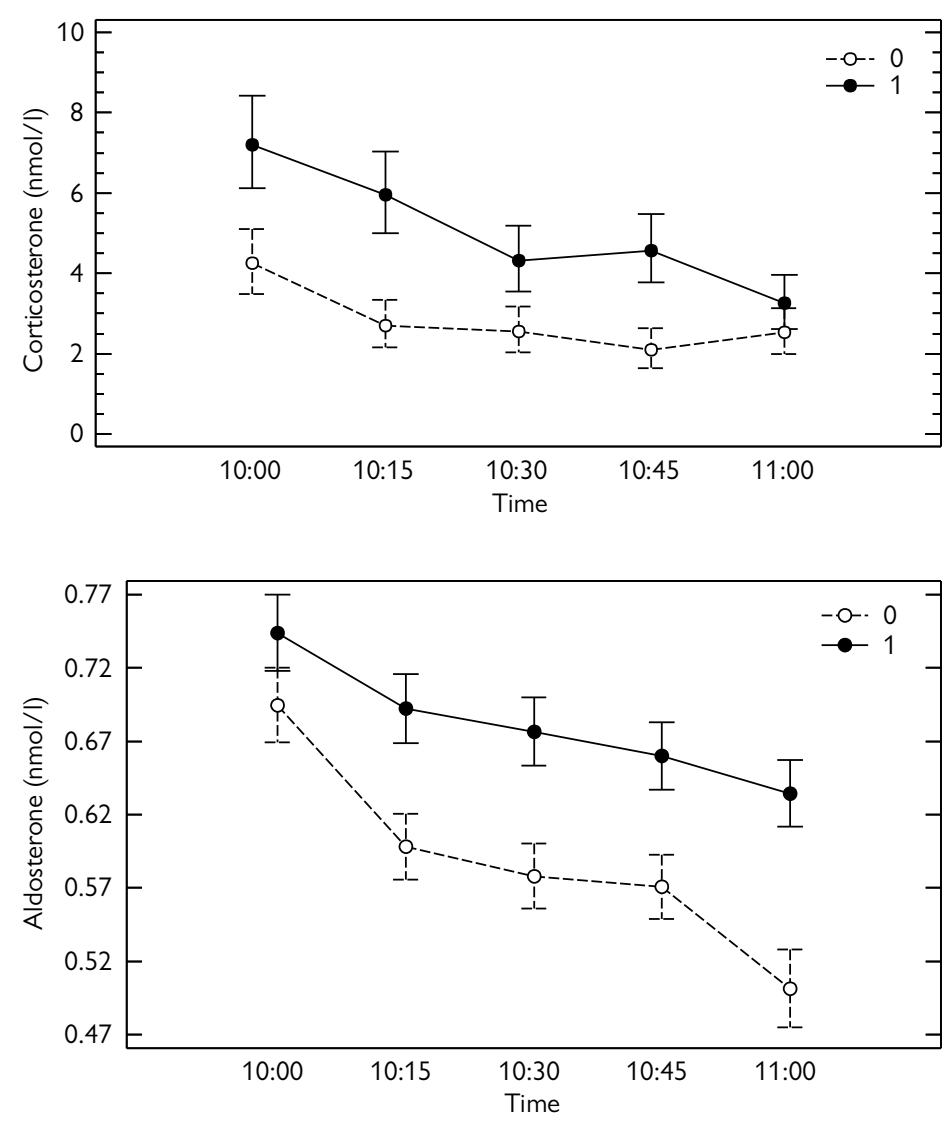

Figure 2 - Influence of the time of blood drawing after cannulisation on levels of corticosterone and aldosterone (0 - cannula inserted at 8:00; 1 - cannula inserted at 10:00).

Corticosterone was also increased already at the time of sampling, while increases in its metabolite aldosterone came later (Figure 2). As opposed to the other corticoids tested, the effect of stress on corticosterone disappeared after an hour (Figures 1 and 2).

The main lunchtime meal

The effects of the main meal of the day - lunch at noontime - were interesting. All corticoids tested had a marked increase between 11 and 12 o'clock, which could reflect a physiological preparation for eating as part of the circadian rhythm. After eating there was an evident decline in cortisone levels, while its precursor cortisol had rather a plateau in its decline (Figure 3). Similarly, there was an evident decline in aldosterone but a plateau in the decline of its precursor corticosterone (Figure 4). 

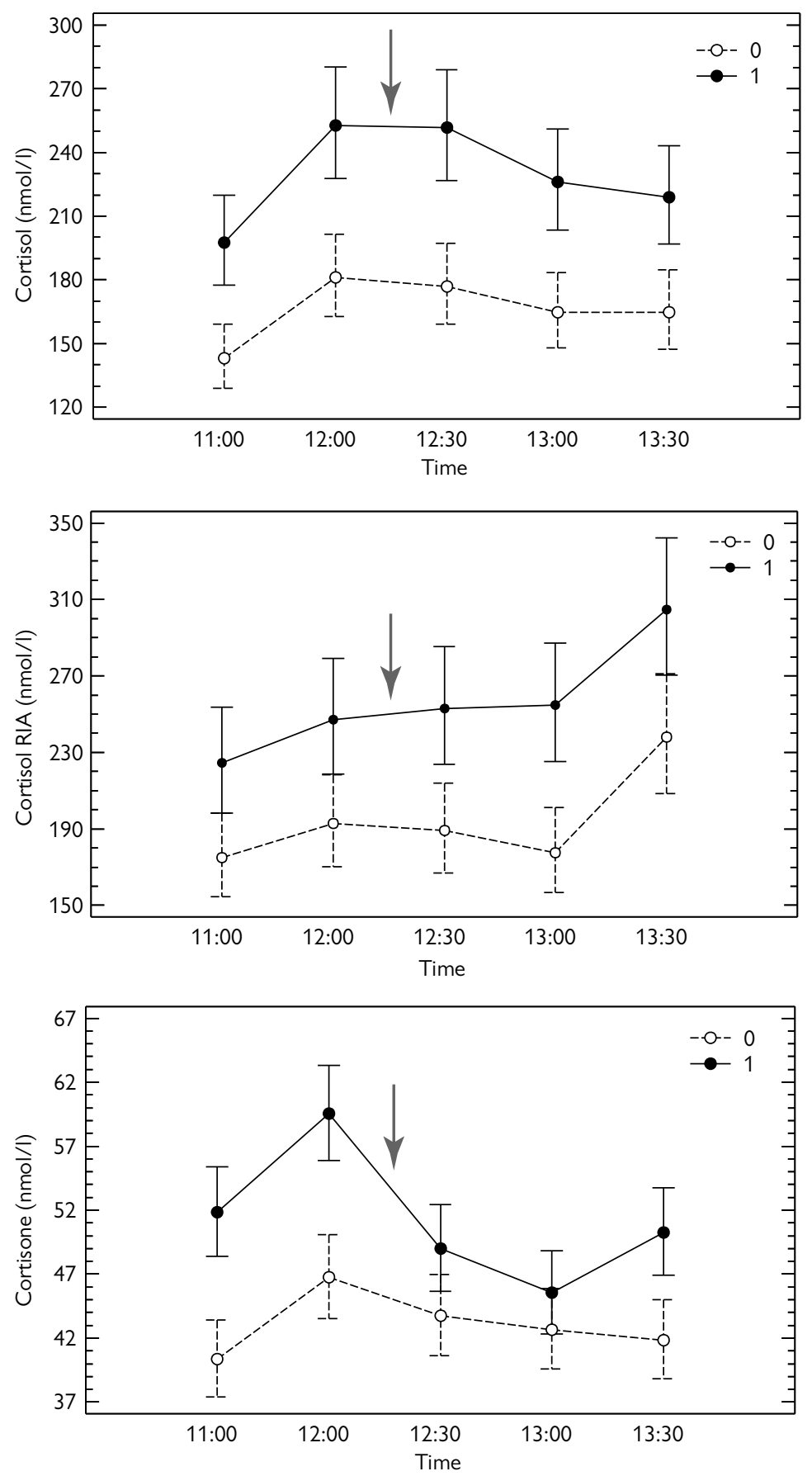

Figure 3 - Cortisol (measured LC-MSIMS and RIA) and cortisone levels before lunch and then at half-hour intervals (0 - with no lunch; 1 - lunch eaten after sampling at 12:00; $\downarrow$-lunch). 

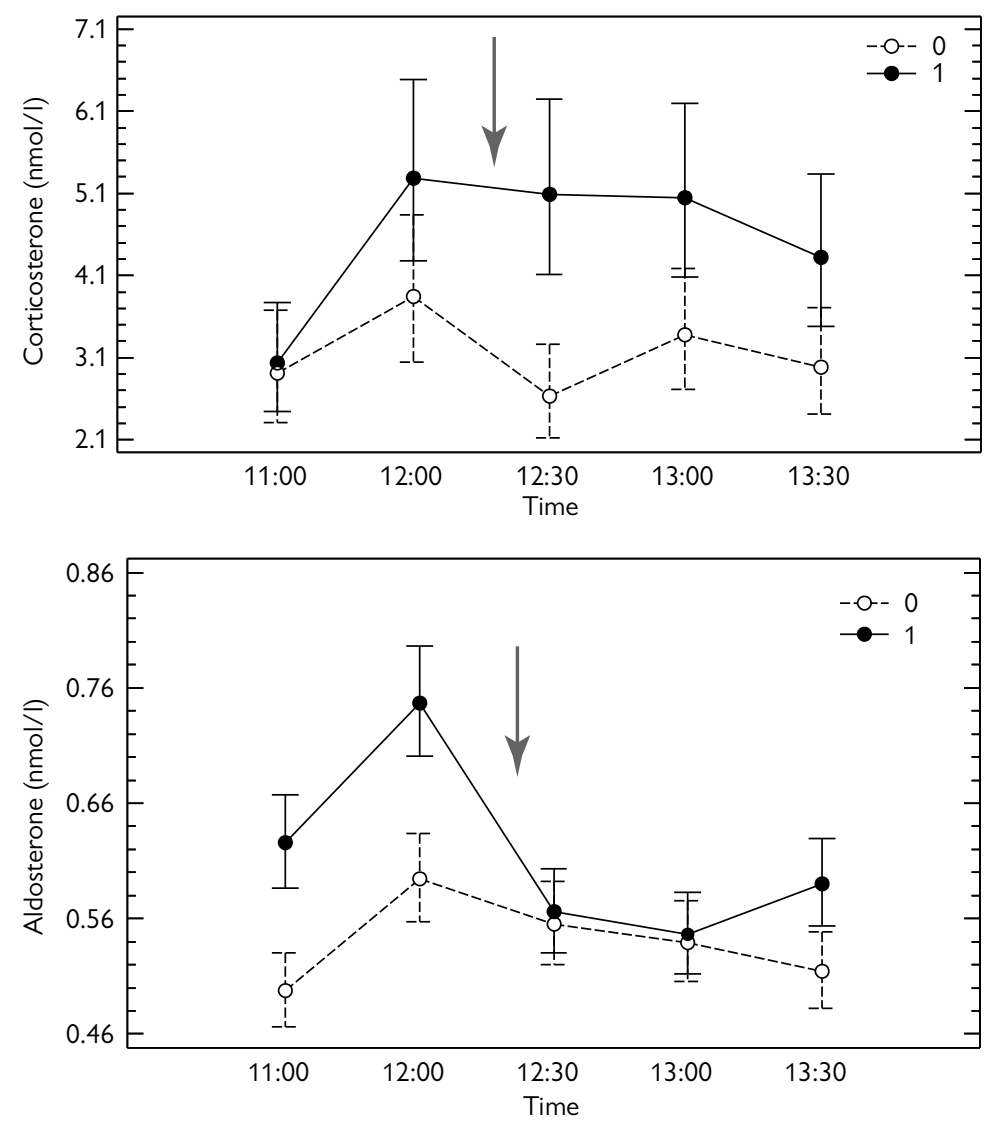

Figure 4 - Levels of corticosterone and aldosterone before lunch and then at half-hour intervals after lunchtime (0 - with no lunch; 1 - lunch eaten after sampling at 12:00; $\downarrow$-lunch).

\section{Choice of analytical method}

We compared cortisol in 90 plasma samples measured by the commercial RIA kit from Immunotech and a published LC-MS/MS method (Sosvorova et al., 2015). The RIA method, which was used as the reference method, showed strong correlation with the $L C$ method $(r=0.85)$, with the regression approximated by the equation $y=0.650 x+49.62$ (Figure 5). The slope of the regression line indicates some overestimation of cortisol levels when using RIA.

\section{Discussion}

In our study we focused on cortisol and its main metabolite cortisone as the primary stress hormones, and on aldosterone as the main mineralocorticoid. We also analysed corticosterone, even though it is a minor glucocorticoid in humans, as opposed to rodents and other vertebrates; it has been hypothesised, however, that 


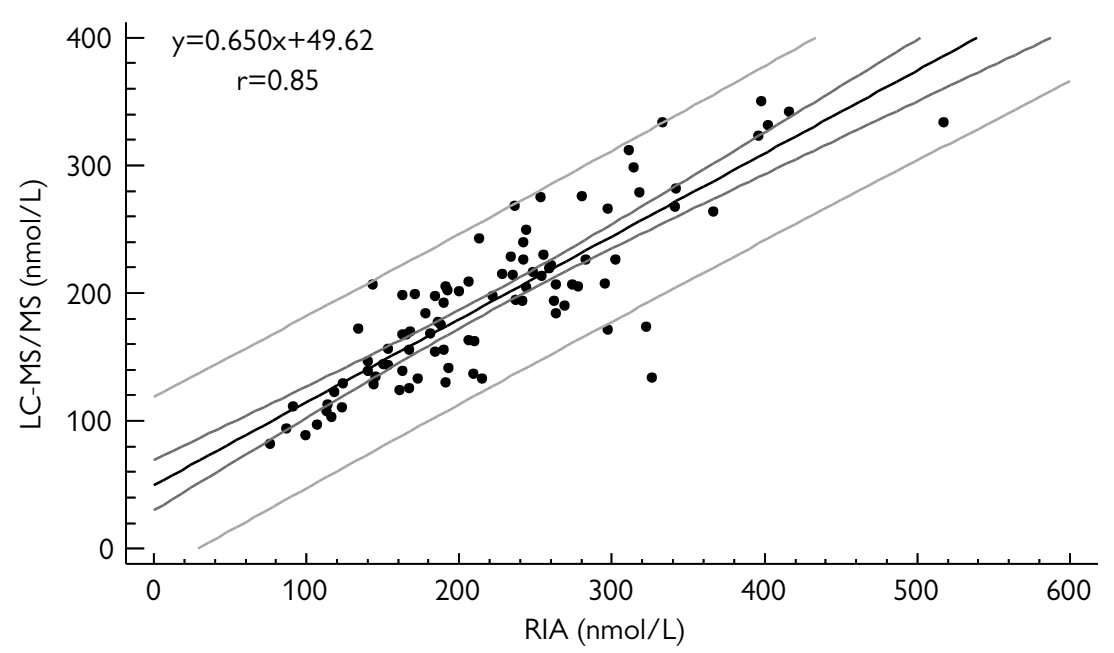

Figure 5 - Comparison of the LC-MSIMS and RIA methods. Simple regression for cortisol. RIA was selected as the reference method and LC-MSIMS as the test method.

under certain physiological conditions corticosterone has additional biochemical functions over and above those of cortisol (Morris, 2015).

Stress is a common reaction to having blood drawn, brought on either directly by the insertion of the needle or even more so by the fear that precedes it. Such emotional excitement can leave traces on physiological functions even two hours after the stimulus abates (Pieper et al., 2007). Here we demonstrate that a stress reaction can precede cannulation and be evident in samples taken during the cannulation procedure. Further increases in cortisol after cannulation were not found, but the subsequent decline was slow and higher levels were found even an hour later.

For a correct interpretation of results, therefore, a calm environment during blood drawing and a sufficiently calm patient are necessary. For clinical studies or research purposes, we recommend taking samples from a cannula that has been inserted 2 hours before the actual blood sampling.

Using salivary cortisol could be completely eliminated the problem of the stress caused by blood sampling. Salivary cortisol represents an easy method and permits possible samplings with high frequency, during the normal activity of patients at home. This method is promising for the future, but needs more data of physiological and normal values (Kosák et al., 2014).

There is as yet little information about the influence of food intake on the daily profile of cortisol. Using 15-minute intervals, Knoll et al. (1984) found higher cortisol levels after lunch compared to after dinner. Goldman et al. (1985) found peak cortisol during both the lunch and dinner periods. The timing of food 
intake is also important (Follenius et al., 1982; Simonetta et al., 1991; Van Cauter et al., 1992; Elimam and Marcus, 2002; Bandin et al., 2015). Studies mostly agree that higher levels occur after food intake, but increases do not occur constantly. Higher cortisol levels after food have even been found in saliva (Toda et al., 2004).

In our study we found physiologically slightly increased cortisol before 12 o'clock, which is the traditional time in the Czech Republic for the main meal (lunch). This could be a physiological reaction to preparing for food intake. This increase was also evident for the other corticoids (cortisone, aldosterone, corticosterone). However, individual corticoids reacted differently to the lunch, with plateaus in the levels of cortisol and corticosterone but continuous declines in the levels of their metabolites (cortisone, respectively aldosterone).

The choice of analytical method

For standard clinical biochemistry, immunoassays are certainly sufficient. It must be kept in mind, however, that in some cases values can be significantly distorted. One example is in women who are taking some types of hormonal contraceptives (Šimůnková et al., 2008). This distortion is partly a result of cross-reactions of antisera for cortisol with products of the metabolism of some gestagens present in combined contraceptive formulas.

For scientific studies and partly for clinical studies it would be more correct to measure cortisol after chromatographic separation, preferably liquid chromatography with mass spectrometry (LC-MS). Values obtained by LC-MS better reflect actual concentrations of circulating cortisol and are statistically significantly lower than values from radioimmunoassay. Another advantage of LC-MS is the possibility to measure multiple steroids in one assay, enabling a more complex perspective. On the other hand, the fact that immunoassays are more accessible for smaller laboratories where mass spectrometry would be non-economical is an argument for the use of RIA. Therefore, with proper standardisation and awareness of the method's limitations, RIA remains the first choice for routine analyses (Taylor et al., 2015).

\section{Conclusion}

Not just endocrinologists but also other specialists often require cortisol measurements from biochemical laboratories. Determining the correct levels of cortisol, especially in the differential diagnosis of hypocortisolism, hypercortisolism and normal functioning of the hypothalamic-pituitary-adrenal axis, require maintaining the proper conditions even in the pre-analytical phase of sampling. It is necessary to take into account the daily rhythm of cortisol and avoid sampling in the first hours after waking (if not specifically measuring CAR), as well as taking into account food intake and the stress of blood drawing. Finally, the choice of proper analytical method should be made with knowledge of their limitations. 


\section{References}

Bandin, C., Scheer, F. A., Luque, A. J., Avila-Gandia,V., Zamora, S., Madrid, J. A., Gomez-Abellan, P., Garaulet, M. (2015) Meal timing affects glucose tolerance, substrate oxidation and circadian-related variables: A randomized, crossover trial. Int. J. Obes. (Lond.) 39(5), 828-833.

Elimam, A., Marcus, C. (2002) Meal timing, fasting and glucocorticoids interplay in serum leptin concentrations and diurnal profile. Eur. J. Endocrinol. 147(2), 181-188.

Follenius, M., Brandenberger, G., Hietter, B. (1982) Diurnal cortisol peaks and their relationships to meals. J. Clin. Endocrinol. Metab. 55(4), 757-761.

Goldman, J., Wajchenberg, B. L., Liberman, B., Nery, M., Achando, S., Germek, O. A. (1985) Contrast analysis for the evaluation of the circadian rhythms of plasma cortisol, androstenedione, and testosterone in normal men and the possible influence of meals. J. Clin. Endocrinol. Metab. 60(1), 164-167.

Knoll, E., Müller, F.W., Ratge, D., Bauersfeld,W., Wisser, H. (1984) Influence of food intake on concentrations of plasma catecholamines and cortisol. J. Clin. Chem. Clin. Biochem. 22(9), 597-602.

Kosák, M., Hána, V., Hill, M., Šimůnková, K., Lacinová, Z., Kršek, M., Marek, J. (2014) Serum cortisol seems to be a more appropriate marker for adrenocortical reserve evaluation in ACTH test in comparison to salivary cortisol. Physiol. Res. 63, 229-236.

Lightman, S. L., Conway-Campbell, B. L. (2010) The crucial role of pulsatile activity of the HPA axis for continuous dynamic equilibration. Nat. Rev. Neurosci. 11(10), 710-718.

Morris, D. J. (2015) Why do humans have two glucocorticoids: A question of intestinal fortitude. Steroids 2(102), 32-38.

Pieper, S., Brosschot, J. F., van der Leeden, R., Thayer, J. F. (2007) Cardiac effects of momentary assessed worry episodes and stressful events. Psychosom. Med. 69, 901-909.

Pruessner, J. C., Wolf, O. T., Hellhammer, D. H., Buske-Kirschbaum, A., von Auer, K., Jobst, S., Kaspers, F., Kirschbaum, C. (1997) Free cortisol levels after awakening: a reliable biological marker for the assessment of adrenocortical activity. Life Sci. 61(26), 2539-2549.

Rácz, B., Dušková, M., Vondra, K., Šrámková, M., Hill, M., Stárka, L. (2015a) Daily profiles of steroid hormones and their metabolites related to food intake. Physiol. Res. 64, S219-S226 (Suppl. 2).

Rácz, B., Dušková, M., Jandíková, H., Hill, M., Vondra, K., Stárka, L. (2015b) How does energy intake influence the levels of certain steroids? Prague Med. Rep. 116(4), 290-302.

Simonetta, G., Walker, D.W., McMillen, I. C. (1991) Effect of feeding on the diurnal rhythm of plasma cortisol and adrenocorticotrophic hormone concentrations in the pregnant ewe and sheep fetus. Exp. Physiol. 76(2), 219-229.

Šimůnková, K., Stárka, L., Hill, M., Kř̌žz, L., Hampl, R., Vondra, K. (2008) Comparison of total and salivary cortisol in a low-dose ACTH (Synacthen) test: influence of three-month oral contraceptives administration to healthy women. Physiol. Res. 57, S193-S199 (Suppl. 1).

Sosvorova, L., Vitku, J., Chlupacova, T., Mohapl, M., Hampl, R. (2015) Determination of seven selected neuro- and immunomodulatory steroids in human cerebrospinal fluid and plasma using MS/MS. Steroids 98, 1-8.

Taylor, A. E., Keevil, B., Huhtaniemi, I. T. (2015) Mass spectrometry and immunoassay: how to measure steroid hormones today and tomorrow. Eur. J. Endocrinol. 173(2), D1-D12.

Toda, M., Morimoto, K., Nagasawa, S., Kitamura, K. (2004) Effect of snack eating on sensitive salivary stress markers cortisol and chromogranin A. Environ. Health Prev. Med. 9(1), 27-29.

Van Cauter, E., Shapiro, E. T., Tillil, H., Polonsky, K. S. (1992) Circadian modulation of glucose and insulin responses to meals: relationship to cortisol rhythm. Am. J. Physiol. 262(4 Pt 1), E467-E475. 